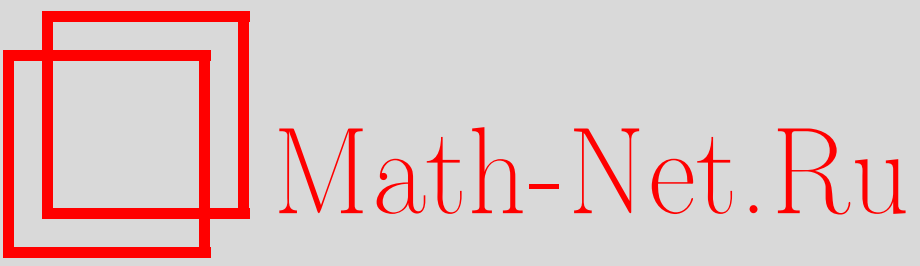

Ф. М. Мухамедов, О эргодическом принципе для марковских процессов, связанных с квантовыми квадратичными стохастическими процессами, УМН, 2002, том 57, выпуск 6, 193-194

DOI: https://doi.org/10.4213/rm588

Использование Общероссийского математического портала Math-Net.Ru подразумевает, что вы прочитали и согласны с пользовательским соглашением

http://www.mathnet.ru/rus/agreement

Параметры загрузки:

IP : 3.93 .64 .190

26 апреля 2023 г., $17: 32: 12$ 


\title{
О ЭРГОДИЧЕСКОМ ПРИНЦИПЕ ДЛЯ МАРКОВСКИХ ПРОЦЕССОВ, СВЯЗАННЫХ С КВАНТОВЫМИ КВАДРАТИЧНЫМИ СТОХАСТИЧЕСКИМИ ПРОЦЕССАМИ
}

\author{
Ф. М. МУХАМЕДОВ
}

В [1] был определен нестационарньй марковский процесс, порождаемый квантовым квадратичным стохастическим процессом (к.к.с.п.), и было отмечено, что если к.к.с.п. удовлетворяет эргодическому принципу, тогда такой марковский процесс также удовлетворяет этому принципу. В настоящей работе с помощью разложения к.к.с.п. (см. [2]) доказьвается, что обратное тоже верно, т.е. если марковский процесс удовлетворяет эргодическому принципу, то соответствующий к.к.с.п. удовлетворяет этому принципу.

Пусть $\mathscr{M}$ - алгебра фон Неймана и $\mathscr{M}_{*}$ - совокупность всех ультраслабо непрерьвных линейных функционалов на $\mathscr{M}$. Через $S$ и $S^{2}$ обозначим совокупности всех нормалшных состояний на $\mathscr{M}$ и $\mathscr{M} \otimes \mathscr{M}$ соответственно. Пусть $\varphi \in S$ - состояние, определим оператор $E_{\varphi}: \mathscr{M} \otimes \mathscr{M} \rightarrow \mathscr{M}$ условного ожидания на элементах вида $a \otimes b, a, b \in \mathcal{M}$, следующим образом: $E_{\varphi}(a \otimes b)=\varphi(a) b$, и далее продолжим по линейности и непрерьвности до $\mathscr{M} \otimes \mathscr{M}$. Ясно, что $E_{\varphi}$ является положительным (т.е. $E_{\varphi} x \geqslant 0$ при $x \geqslant 0$ ) и $E_{\varphi} \mathbf{1} \mathscr{M}=\mathbf{1} \mathscr{M} \otimes \mathscr{M}$, где $\mathbf{1}_{\mathscr{M}}$ и $\mathbf{1} \mathscr{M} \otimes \mathscr{M}$ - единицы алгебр $\mathscr{M}$ и $\mathscr{M} \otimes \mathscr{M}$ соответственно.

Пусть $\left\{P^{s, t}: \mathscr{M} \rightarrow \mathscr{M} \otimes \mathscr{M}, s, t \in \mathbb{R}_{+}, t-s>1\right\}-$ семейство линейных операторов и $U: \mathscr{M} \otimes \mathscr{M} \rightarrow \mathscr{M} \otimes \mathscr{M}$ - линейньй оператор такой, что $U(x \otimes y)=y \otimes x$ для всех $x, y \in \mathscr{M}$.

ОПредЕЛЕнИЕ 1. Будем говорить, что пара $\left(P^{s, t}, \omega_{0}\right)$, где $\omega_{0} \in S$ - началњное состояние, образует квантовый квадратичный стохастический прочесс (к.к.с.п.), если каждьй оператор $P^{s, t}$ ультраслабо непрерьвен и при этом выполняются следующие соотношения:

(i) $P^{s, t} \mathbf{1}_{\mathscr{M}}=\mathbf{1} \mathscr{M} \otimes \mathscr{M}$;

(ii) $P^{s, t}\left(\mathscr{M}_{+}\right) \subset(\mathscr{M} \otimes \mathscr{M})_{+}$;

(iii) $U P^{s, t} x=P^{s, t} x$ для любого $x \in \mathscr{M}$;

(iv) аналог уравнения Колмогорова-Чепмена: для произвольных $s, \tau, t \in \mathbb{R}_{+}$таких, что $\tau-s \geqslant 1, t-\tau \geqslant 1$

либо

(iv) ${ }_{\mathrm{A}} P^{s, t} x=P^{s, \tau}\left(E_{\omega_{\tau}}\left(P^{\tau, t} x\right)\right), x \in \mathscr{M}$, либо

(iv) ${ }_{\mathrm{B}} P^{s, t} x=E_{\omega_{s}} P^{s, \tau} \otimes E_{\omega_{s}} P^{s, \tau}\left(P^{\tau, t} x\right), x \in \mathscr{M}$, где $\omega_{\tau}(x)=\omega_{0} \otimes \omega_{0}\left(P^{0, \tau} x\right), x \in \mathscr{M}$.

Если к.к.с.п. удовлетворяет равенству (iv) А или (iv) В, то будем говорить, что к.к.с.п. имеет тип (А) или тип (В) соответственно.

Пусть $\mathscr{T}=\left\{T^{s, t}(\varphi): \mathscr{M} \rightarrow \mathscr{M} \mid s, t \in \mathbb{R}_{+}, t-s \geqslant 1, \varphi \in \mathscr{M}_{*}\right\}$ - семейство улштраслабо непрерывных линейных отображений на алгебре фон Неймана $\mathscr{M}$.

ОПредЕЛЕниЕ 2 . Пара $\left(\mathscr{T}, \omega_{0}\right)$, где $\omega_{0} \in S$-начальное состояние, назьвается слойно-марковским процессом (с.м.п.), если при фиксированных значениях $s$ и $t T^{s, t}(\cdot)$ является линейной функцией на $\mathscr{M}_{*}$ и выполнены следующие условия:

(i) $T^{s, t}(\varphi) \mathbf{1}_{\mathscr{M}}=\mathbf{1}_{\mathscr{M}}$ при $\varphi \in S$;

(ii) $T^{s, t}(\varphi)\left(\mathscr{M}_{+}\right) \subset \mathscr{M}_{+}$при $\varphi \in \mathscr{M}_{*,+}$;

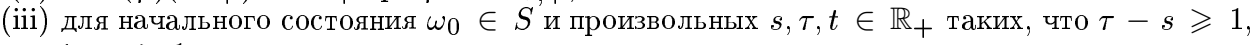
$t-\tau \geqslant 1$

либо

(iii) $T^{s, t}(\varphi)=T^{s, \tau}(\varphi) T^{\tau, t}\left(\omega_{\tau}\right), \varphi \in S$, либо

$(\mathrm{iii})_{\mathrm{B}} T^{s, t}(\varphi)=T^{s, \tau}\left(\omega_{s}\right) T^{\tau, t}\left(T_{*}^{s, \tau}\left(\omega_{s}\right) \varphi\right), \varphi \in S$, где $\omega_{\tau}(x)=\omega_{0}\left(T^{0, \tau}\left(\omega_{0}\right) x\right), x \in \mathscr{M}$, и $\left(T_{*}^{s, t}\left(\omega_{s}\right) \varphi\right)(x)=\varphi\left(T^{s, t}\left(\omega_{s}\right) x\right), x \in \mathscr{M}$. 
Будем говорить, что с.м.п. ( $\left.\mathscr{T}, \omega_{0}\right)$ имеет тип $($ А) или тип $($ В) соответственно, в зависимости от того, какое из фундаментальных уравнений (iii) А или $(\mathrm{iii})_{\mathrm{B}}$ имеет место. С.м.п. $\left(\mathscr{T}, \omega_{0}\right)$ называется симметричным, если для любых $\varphi, \psi \in S$ имеет место $T_{*}^{s, t}(\varphi) \psi=T_{*}^{s, t}(\psi)_{*} \varphi$.

Tеорема 1. Каждому к.к.c.n. $\left(P^{s, t}, \omega_{0}\right)$ muna (A) (соответственно типа (B)) однозначно соответствует симметричный с.м.n. ( $\left.\mathscr{T}_{p}, \omega_{0}\right)$ типа (A) (соответственно типа (В)), определенный следующим образом:

$$
T^{s, t}(\varphi) x=E_{\varphi}\left(P^{s, t} x\right), \quad \varphi \in \mathscr{M}_{*}, \quad x \in \mathscr{M} .
$$

Обратно, каждому симметричному с.м.n. $\left(\mathscr{T}_{p}, \omega_{0}\right)$ однозначно соответствует א.к.c.n. $\left(P^{s, t}, \omega_{0}\right)$ соответствующего типа, для которого имеет место (1).

Через $P_{*}^{s, t}$ обозначим оператор, действующий из $S^{2}$ в $S$, определенный следующим образом: $P_{*}^{s, t}(\varphi)(x)=\varphi\left(P^{s, t} x\right)$, где $\varphi \in S^{2}, x \in \mathscr{M}$. Будем говорить, что к.к.с.п. $\left(P^{s, t}, \omega_{0}\right)$ удовлетворяет әргодическому принципу, если для любых $\varphi, \psi \in S^{2}$ и $s \in \mathbb{R}_{+}$имеет место

$$
\lim _{t \rightarrow \infty}\left\|P_{*}^{s, t} \varphi-P_{*}^{s, t} \psi\right\|_{1}=0,
$$

где $\|\cdot\|_{1}-$ норма на $\mathscr{M}^{*}$

Отметим, что понятие эргодического принципа было введено впервые для марковского процесса в работах Колмогорова (см., например, [3]), а для квадратичных процессов в [1], [4].

Будем говорить, что с.м.п. $\left(\mathscr{T}, \omega_{0}\right)$ удовлетворяет условию $(\mathrm{E})$, если существует число $\lambda \in$ $[0,1)$ такое, что для любых $\varphi, \psi, \sigma \in S$ и $s \in \mathbb{R}_{+}$неравенство $\left\|T_{*}^{s, t}(\sigma) \varphi-T_{*}^{s, t}(\sigma) \psi\right\|_{1} \leqslant$ $\lambda\|\varphi-\psi\|_{1}$ выполнено хотя бы для одного $t=t(\varphi, \psi, \sigma, s) \in \mathbb{R}_{+}$.

Определим новый процесс $Q^{s, t}: \mathscr{M} \rightarrow \mathscr{M}$ следующим образом: $Q^{s, t} x=T^{s, t}\left(\omega_{s}\right) x, x \in \mathscr{M}$.

ПреДЛоЖение 1. Пусть $P^{s, t}-$ א.к.c.n. на алгебре фон Неймана $\mathscr{M}$ u $\left(\mathscr{T}, \omega_{0}\right)-$ coоmветствующий ему с.м.п. Тогда $Q^{s, t}$ является марковским процессом, т.е. для любих чисел $s, \tau, t \in \mathbb{R}_{+}$таких, что $\tau-s \geqslant 1, t-\tau \geqslant 1$, имеет место равенство $Q^{s, t}=Q^{s, \tau} Q^{\tau, t}$.

Теорема 2. Пусть $P^{s, t}-$ к.к.c.n. на алгебре фон Неймана $\mathscr{M}$ и $\left(\mathscr{T}, \omega_{0}\right)$ - его c.м.n.разложение. Тогда следующие условия әквивалентны:

(i) $P^{s, t}$ удовлетворяет эргодическому принципу;

(ii) c.м.n. $\left(\mathscr{T}, \omega_{0}\right)$ удовлетворяет условию $(\mathrm{E})$;

(iii) марковский процесс $Q^{s, t}$ удовлетворяет әргодическому принципу.

Разложение к.к.с.п. позволяет нам определить понятия сопряженности двух к.к.с.п., определенных на алгебре фон Неймана, следующим образом.

Пусть $\left(P^{s, t}, \omega_{0}\right)$ и $\left(H^{s, t}, \omega_{0}\right)$ - два к.к.с.п. на алгебре фон Неймана $\mathscr{M}$, и пусть $\left(\mathscr{T}_{P}, \omega_{0}\right)$ и $\left(\mathscr{T}_{H}, \omega_{0}\right)-$ их разложения на с.м.п. соответственно. Будем говорить, что к.к.с.п. $\left(P^{s, t}, \omega_{0}\right)$ и $\left(H^{s, t}, \omega_{0}\right)$ сопряжень, и обозначать $P^{s, t} \sim H^{s, t}$, если существует семейство автоморфизмов $\left\{\theta^{s, t}(\varphi): s, t \in \mathbb{R}_{+}, t-s \geqslant 1, \varphi \in \mathscr{M}_{*}\right\}$ алгебры $\mathscr{M}$ в себя такое, что для любых чисел $s, t \in \mathbb{R}_{+}, t-s \geqslant 1$, и функционала $\varphi \in \mathscr{M}_{*}$ имеет место равенство $T_{P}^{s, t}(\varphi) \theta^{s, t}(\varphi)=T_{H}^{s, t}(\varphi)$.

Tеорема 3. Пусть $\left(P^{s, t}, \omega_{0}\right) u\left(H^{s, t}, \omega_{0}\right)$ - сопряженные к.к.с.п. на алгебре фон Неймана $\mathscr{M}$. Тогда $\left(P^{s, t}, \omega_{0}\right)$ удовлетворяет эргодическому принципу тогда и только тогда, когда $\left(H^{s, t}, \omega_{0}\right)$ удовлетворяет әргодическому приниипу.

\section{СПИСОК ЛИТЕРАТУРЫ}

[1] Н. Н. Ганиходжаев, Ф. М. Мухамедов // Изв. РАН, сер. матем. 2000. Т. 65. № 5. С. 3-20. [2] Ф. М. Мухамедов // Докл. РАН. 2000. Т. 371. № 2. С. 167-169. [3] А. Н. Колмогоров // УМН. 1938. № 5. С. 5-51. [4] Т. А. Сарымсаков, Н. Н. Ганиходжаев // Докл. AH CCCP. 1991. T. 316. № 6. C. 1315-1319. 